\title{
Mecanismos inflamatorios involucrados en la fisiopatología de la hiperplasia prostática benigna
}

\section{Inflammatory Mechanisms Involved in the Pathophysiology of Benign Prostatic Hyperplasia}

\author{
Ximena Guzman Robledo ${ }^{1}$ Herney Andrés García-Perdomo ${ }^{2}{ }^{\circ}$ \\ 1 Universidad del Valle, Cali, Colombia \\ ${ }^{2}$ Escuela de Medicina, Grupo de Investigación UROGIV, Universidad \\ del Valle, Cali, Colombia \\ Address for correspondence Herney Andrés García-Perdomo, MD, \\ MSc, EdD, PhD, FACS, Escuela de Medicina, Grupo de Investigación \\ UROGIV, Universidad del Valle, Dirección: Calle 4B \#36-00, Cali, \\ Colombia (e-mail: herney.garcia@correounivalle.edu.co).
}

Urol Colomb 2020;29:240-244.

\section{Resumen \\ Palabras Clave \\ - hiperplasia prostática benigna \\ - obstrucción del tracto de salida \\ - inflamación crónica}

La obstrucción del tracto de salida ha sido asociada con la hiperplasia prostática benigna (HPB), dado el crecimiento progresivo del adenoma, lo cual lleva a un incremento en la resistencia al flujo urinario, junto con un efecto deletéreo en la función renal. Se han descrito múltiples teorías asociadas con el desarrollo de la hiperplasia. Entre las cuales se encuentran: La edad, el incremento en la actividad simpática, alteraciones hormonales, el síndrome metabólico y la inflamación crónica. En los últimos años, el rol de la inflamación prostática local en el entendimiento de la patogénesis y progresión de la HPB ha cobrado relevancia. Esa hipótesis plantea que infiltrados inflamatorios conllevan a una lesión tisular, generando así un proceso crónico de cicatrización que condiciona el incremento en el tamaño prostático. El presente artículo se enfoca en describir los mecanismos inflamatorios involucrados en la fisiopatología de la hiperplasia prostática benigna.

Urinary tract obstruction has been associated with benign prostatic hyperplasia (BPH), given the progressive adenoma growth. This process leads to an increase in urinary flow resistance, associated with a deleterious effect of renal function. Age, increased sympathetic activity, hormonal alterations, metabolic syndrome and chronic inflammation are among the multiple theories associated with the development of hyperplasia. In recent years, the role of local prostatic inflammation in understanding the pathogenesis and progression of BPH has become relevant. This hypothesis suggests that inflammatory infiltrates lead to a tissue injury, thus generating a chronic healing process that conditions the prostatic increase. The present article focuses on describing the inflammatory mechanisms involved on BPH pathophysiology.

\section{Introducción}

La hiperplasia prostática benigna (HPB), es la enfermedad urológica ligada a la edad más frecuente. Consiste en un

received

April 19, 2019

accepted

December 2, 2019
DOI https://doi.org/

10.1055/s-0040-1701278.

ISSN 0120-789X.

e ISSN 2027-0119. incremento progresivo de la próstata por un proceso proliferativo no maligno del componente epitelial y estromal de la zona transicional y periuretral de la glándula prostática. ${ }^{1-3}$
Copyright (c) 2020, Sociedad Colombiana License terms de Urología. Publicado por Thieme Revinter Publicações Ltda., Rio de Janeiro, Brazil. Todos los derechos reservados. 
Ese crecimiento se asocia con un aumento en la resistencia al flujo de salida con efectos secundarios sobre la función vesical y renal y se manifiesta clínicamente en la forma de síntomas del tracto urinario bajo (STUB). Los cuales pueden ser de vaciamiento como chorro urinario débil, chorro en regadera, intermitencia, vacilación, pujo y goteo terminal; asî como de almacenamiento incluyendo un aumento en la frecuencia urinaria, urgencia y nocturia. ${ }^{3,4}$

A pesar que múltiples teorías han sido propuestas para explicar el mecanismo subyacente de la HPB, la patogénesis aún no es entendida completamente. La contribución del crecimiento prostático en el desarrollo de STUB es inequívoco, sin embargo, estudios modernos han demostrado que el desarrollo de los síntomas es multifactorial., ${ }^{2,3}$

El envejecimiento, las alteraciones hormonales, los ambientes proinflamatorios (resistencia a la insulina, hiperinsulinemia secundaria y síndrome metabólico), el aumento en la actividad simpática y la inflamación prostática local son algunos de los factores involucrados en la fisiopatología de la HPB. Recientemente, esa última se ha encontrado asociada además, con la severidad de los síntomas y progresión de la enfermedad. ${ }^{1}$

\section{Teorías implicadas en el desarrollo de la HPB \\ Edad}

Como ya se mencionó previamente, la edad representa el factor de riesgo más importante en el desarrollo de la HPB y por tanto de los STUB. Durante el envejecimiento, se produce interferencia en las vías de los factores de crecimiento, generando un proceso de remodelación tisular que conlleva al crecimiento prostático. ${ }^{5}$

\section{Aumento en la actividad nerviosa simpática}

Una porción sustancial de la próstata está conformada por músculo liso y se cree que posee propiedades contráctiles similares a otros órganos con ese tipo de músculo. Se ha reportado que el sistema nervioso adrenérgico regula el tono del músculo liso, siendo el subtipo $\alpha 1 \mathrm{~A}$ el adrenoreceptor más común presente en el tejido prostático y el encargado de mediar la tensión activa. Estudios previos han demostrado que la densidad estromal de ese tipo de receptores está aumentada. Por lo tanto, la estimulación de esos receptores resulta en un aumento dinámico de la resistencia en la uretra prostática, lo cual se traduce en síntomas obstructivos al flujo de salida. ${ }^{5,6}$

\section{Alteraciones hormonales}

La enzima esteroidea $5 \alpha$-reductasa 2 , la cual está unida a la membrana nuclear, convierte la testosterona en dihidrotestosterona (DHT), el principal andrógeno en la próstata correspondiendo al $90 \%$ de los andrógenos prostáticos totales. $^{6}$ El estroma y el epitelio prostático interactúan a través de mecanismos de señalización celular mediados por DHTy factores de crecimiento dependientes de DHT. Las investigaciones animales y humanas sugieren que el desarrollo de la HPB implica la alteración de la homeostasis mediada por la DHT entre la proliferación y la muerte celular, permitiendo que los procesos proliferativos predominen. ${ }^{6}$ Los factores de crecimiento estimulados por la DHT incluyen el Factor de Crecimiento Epidérmico (EGF), Factor de Crecimiento de Keratinocitos (KGF) y el Factor de Crecimiento similar a la Insulina (IGFs), los cuales modulan la proliferación celular en la próstata humana. Asimismo, la expresión del Factor de Crecimiento Transformante tipo $\beta$ (TGF $\beta$ ), que modula la apoptosis, también está influenciada por la DHT. 6

No obstante, más que un aumento en los niveles o actividad de los factores de crecimiento, es la interacción entre ellos y las hormonas esteroideas lo que puede alterar el balance entre la proliferación y la apoptosis. En efecto, se ha encontrado que tanto en hombres con o sin HPB, las concentraciones intraprostáticas de DHT se mantienen constantes independientemente de la edad, lo cual respalda esa idea. ${ }^{6}$

\section{Síndrome metabólico}

El Síndrome Metabólico (SM) describe un conjunto de múltiples anormalidades metabólicas incluyendo obesidad central, dislipidemia, hipertensión arterial, intolerancia a la glucosa, y resistencia a la insulina e hiperinsulinemia compensatoria secundaria. ${ }^{7}$ Se ha visto directamente asociado con un aumento en el riesgo de enfermedad coronaria, enfermedad cardiovascular aterosclerótica y diabetes mellitus tipo 2 (DM-2). Estudios epidemiológicos, clínicos e histopatológicos recientes aportan evidencia suficiente acerca del posible rol del síndrome metabólico en la patogénesis de la HPB y cáncer de próstata (CaP). ${ }^{8,9}$

En la mayoría de los casos, el SM es la consecuencia de malos hábitos alimenticios y/o estilos de vida sedentarios. La resistencia a la insulina ocurre cuando se genera una disminución en la respuesta de los tejidos periféricos (músculo esquelético, tejido adiposo e hígado), a la acción de la insulina con una hiperinsulinemia concomitante la cual es responsable de la producción del Factor de Crecimiento Similar a la Insulina (IGF-1) en el hígado, que posee afinidad por el receptor de insulina y funciona como un potente factor mitogénico y antiapoptótico, que también se ha encontrado ligado con el riesgo de CaP. Además, a medida que la insulina aumenta, la proteína ligadora de IGF-1 disminuye, de manera tal que se aumenta la biodisponibilidad del IGF-1 ( - Figura 1). ${ }^{7,8}$

Asimismo, la hiperglicemia con hiperinsulinemia puede aumentar los niveles de calcio libre en el citosol de las células musculares lisas y el tejido neural, generando un aumento en la actividad del sistema nervioso simpático, incrementando la severidad de los STUB. ${ }^{8}$

Con respecto a la obesidad central, el tejido adiposo visceral secreta adipoquinas que inducen resistencia a la insulina con efectos proinflamatorios y aterogénicos. La leptina, la resistina, el Factor de Necrosis Tumoral Alfa (TNF- $\alpha$ ), la Interleuquina (IL) 6,8 y $1 \beta$ la Proteína C Reactiva (CRP), el fibrinógeno y el Inhibidor del Activador del Plasminógeno 1 (PAI-1), se encuentran típicamente elevados en pacientes obesos y diabéticos. $8,10,11$

Ese ambiente inflamatorio observado en pacientes con SM juega un papel importante en el desarrollo de la HPB. La 


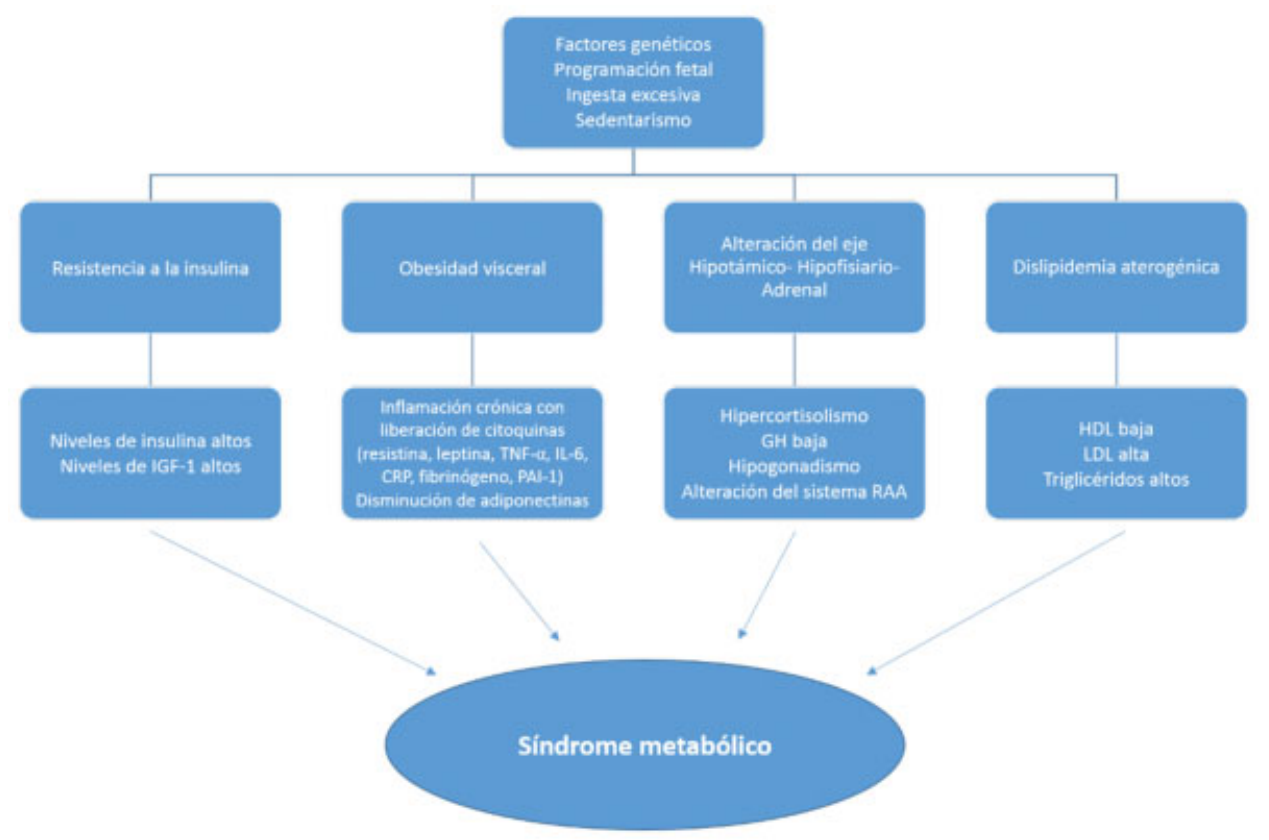

IGF-1 $=$ Factor de Crecimiento Similar a la Insulina; TNF- $\alpha=$ Factor de Necrosis Tumoral Alfa; IL-6= Interleuquina 6; CRP= Proteína C Reactiva; PAI-1= Inhibidor del Activador del Plasminógeno 1; GH=Hormona del crecimiento; RAA=Renina Angiotensina Aldosterona; $\mathrm{HDL}=$ Lipoproteína de alta densidad; LDL: Lipoproteína de baja densidad

Fig. 1 Fisiopatología del síndrome metabólico.

actividad de los linfocitos $\mathrm{T}$ en los infiltrados inflamatorios prostáticos resulta en la estimulación de la proliferación del tejido estromal y epitelial. La lesión tisular y el proceso crónico de cicatrización inducido por la inflamación conducen a la formación de nódulos prostáticos. ${ }^{8}$

Las hormonas sexuales pueden contribuir también a esa asociación. Los hombres con HPB y STUB a menudo se presentan con alteraciones en la relación de andrógenos y estrógenos, y se ha demostrado que los estrógenos sinergizan los efectos de los andrógenos ocasionando un aumento cuatro veces mayor en el volumen prostático total. ${ }^{12,13}$

Hipótesis alternativas atribuyen los STUB a alteraciones funcionales a nivel de la próstata y la vejiga secundarias a un proceso de isquemia crónica derivado de aterosclerosis pélvica (-Figura 2). ${ }^{8,14}$

\section{Inflamación prostática crónica}

La próstata es un órgano inmunocompetente poblado por un pequeño número de células inflamatorias. La respuesta inmune está mediada principalmente por linfocitos $\mathrm{T}$ que corresponden al $90 \%$ de los linfocitos prostáticos totales, y se encuentran tanto en el epitelio como en el estroma. ${ }^{5,15} \mathrm{La}$ inflamación prostática confirmada histológicamente es un hallazgo habitual en biopsias y especímenes quirúrgicos de hombres con HPB, y se reporta hasta en un $44-77 \%$ de las muestras. La inflamación crónica, se encuentra presente en el $78 \%$ de los casos, en comparación con la inflamación aguda en el $15 \%$, $^{1,3}$

Asimismo, los pacientes con HPB e inflamación crónica, tienden a tener un mayor volumen prostático, STUB más severos, son más propensos a desarrollar retención urinaria aguda y tienen una peor respuesta al tratamiento médico convencional respecto a aquellos sin presencia de inflamación. ${ }^{1,16}$ El origen de esa inflamación crónica aún es incierto, no obstante se cree que reside en la presencia de múltiples estímulos concomitantes. Diferentes fuentes de inflamación han sido propuestas, incluyendo infecciones bacterianas (Escherichia coli), virales (Virus del Papiloma Humano, Virus del Herpes Simplex y Citomegalovirus), microorganismos de transmisión sexual (Neisseria gonorrhea, Treponemapallidum, Chlamydia trachomatis y Trichomonas vaginalis), factores relacionados con la dieta, cambios en los niveles de estrógenos y testosterona, respuesta autoinmune, reflujo urinario y alteraciones metabólicas. $^{3,5}$ La persistencia de uno, o la combinación de dos o más de esos estímulos, puede conducir al desarrollo de la inflamación prostática crónica. ${ }^{5,15,16}$

La inflamación crónica y los cambios endocrinos conllevan a la alteración en la homeostasis y a la lesión tisular, mediante la liberación de citoquinas y el aumento en la concentración de factores de crecimiento que interactúan con señales paracrinas en el estroma y epitelio regulando el crecimiento prostático. Esas citoquinas incluyen la IL-1, IL6, IL-8, IL-15, IL-17, Interferón gamma (IFN-[ฺ), Factor de crecimiento transformante $\beta$ (TGF- $\beta$ ), Factor de crecimiento fibroblástico 2 y 7 (FGF 2 y 7) y Factor de crecimiento insulínico 1 (IGF-1). Toda esa cascada de eventos se traduce en una proliferación celular compensatoria y alteraciones en la homeostasis que inducen a su vez una inflamación crónica y cambios endocrinos. ${ }^{2,3,5}$ De cualquier forma, se 


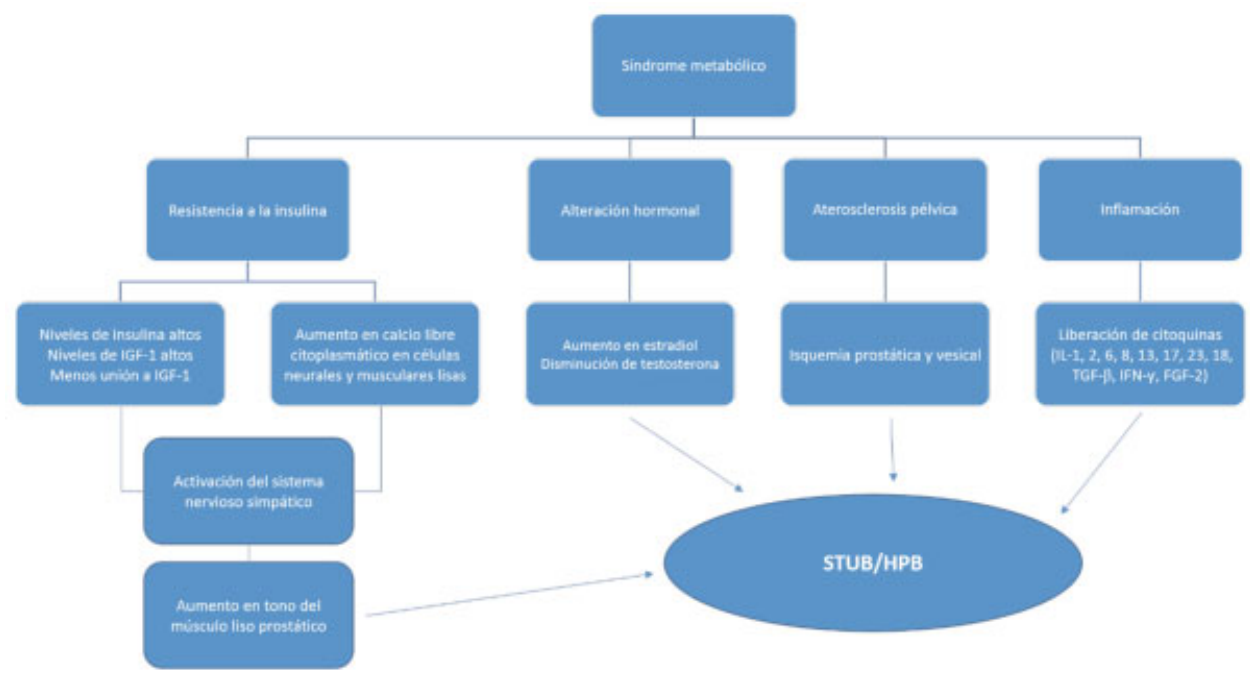

IGF- $1=$ Factor de Crecimiento Similar a la Insulina; $\mathrm{IL}=$ Interleuquina; $\mathrm{TGF}-\beta=$ Factor de Crecimiento Transformante Beta; IFN- $y=$ Interferón gama; FGF-2=Factor de Crecimiento fibroblástico 2; STUB=Sintomas de tracto urinario bajo; HPB=Hiperplasia prostática benigna

Fig. 2 Mecanismos biológicos para síntomas del tracto urinario bajos e hiperplasia prostática benigna en relación al síndrome metabólico.

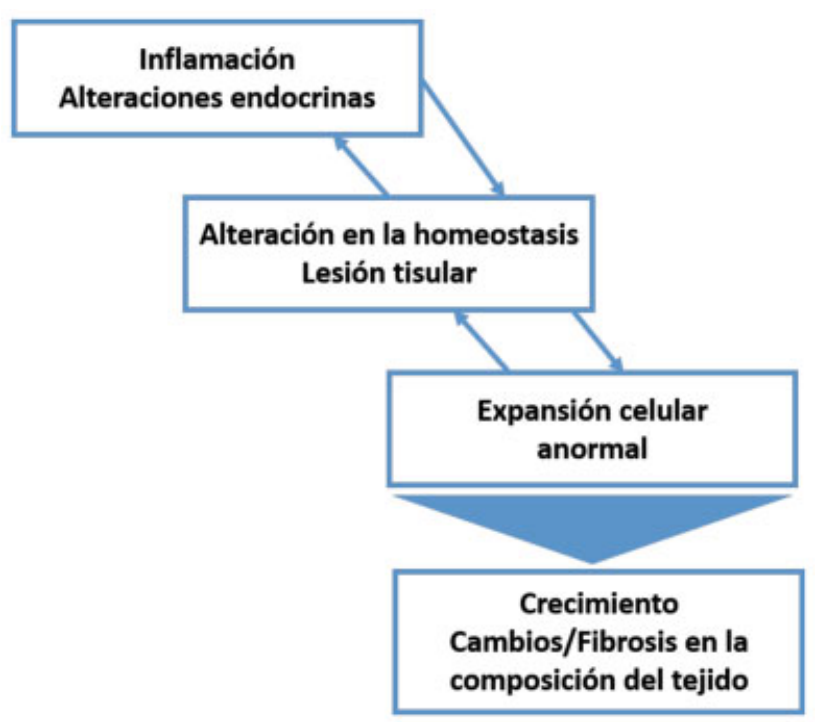

Fig. 3 La inflamación como parte de un "círculo vicioso" de cambios glandulares que genera alteraciones en la arquitectura y volumen prostático.

inicia un círculo vicioso que contribuye al desarrollo de hiperplasia con fibrosis (- Figura 3$)^{2,15}$

\section{Conclusión}

La presencia de inflamación podría explicar algunas de las incertidumbres en los modelos de la HPB desarrollados hasta la fecha. Adicionalmente, en la fisiopatología de la HPB, se presume que la inflamación prostática crónica puede ser tanto un evento iniciador como una consecuencia, y formar parte de un efecto dominó sin embargo, aun no es claro su papel en el proceso.

\section{Conflicto de intereses}

Los autores declaran no tener ningún conflicto de intereses.

\section{Referencias}

1 Burgos J. Inflammation and Prostatic Diseases: From Bench to Bedside. Eur Urol Suppl 2015;14(09):e1453-e1454

2 Schalken JA. Inflammation in the Pathophysiology of Benign Prostatic Hypertrophy. Eur Urol Suppl 2015;14(09):e1455-e1458

3 Bushman WA, Jerde TJ. Role of Prostate Inflammation and Fibrosis in Benign Prostate Hyperplasia and Lower Urinary Tract Symptoms. Am J Physiol Ren Physiol 2016

4 Abrams P, Cardozo L, Fall M, et al; Standardisation Sub-Committee of the International Continence Society. The standardisation of terminology in lower urinary tract function: report from the standardisation sub-committee of the International Continence Society. Urology 2003;61(01):37-49

5 Gandaglia G, Briganti A, Gontero P, et al. The role of chronic prostatic inflammation in the pathogenesis and progression of benign prostatic hyperplasia (BPH). BJU Int 2013;112(04):432-441

6 Chughtai B, Forde JC, Thomas DDM, et al. Benign prostatic hyperplasia. Nat Rev Dis Primers 2016;2:16031

7 Ngai H-Y, Yuen KS, Ng C-M, Cheng C-H, Chu SP. Metabolic syndrome and benign prostatic hyperplasia: An update. Asian J Urol 2017;4(03):164-173

8 De Nunzio C, Aronson W, Freedland SJ, Giovannucci E, Parsons JK. The correlation between metabolic syndrome and prostatic diseases. Eur Urol 2012;61(03):560-570

9 Yang T-K, Hsieh J-T, Chen S-C, Chang H-C, Yang H-J, Huang K-H. Metabolic syndrome associated with reduced lower urinary tract symptoms in middle-aged men receiving health checkup. Urology 2012;80(05):1093-1097

10 Liu L, Li Q, Han P, et al. Evaluation of interleukin-8 in expressed prostatic secretion as a reliable biomarker of inflammation in benign prostatic hyperplasia. Urology 2009;74(02):340-344

11 Alexandraki K, Piperi C, Kalofoutis C, Singh J, Alaveras A, Kalofoutis A. Inflammatory Process in Type 2 Diabetes: The Role of Cytokines. Ann N Y Acad Sci 2006;1084(01):89-117 


\section{Inflamación en hiperplasia prostática benigna Robledo, García-Perdomo}

12 Asiedu B, Anang Y, Nyarko A, et al. The role of sex steroid hormones in benign prostatic hyperplasia. Aging Male 2017;20 (01):17-22

$13 \mathrm{Wu}$ Y, Pan $\mathrm{H}$, Wang W-M, et al. A possible relationship between serum sex hormones and benign prostatic hyperplasia/ lower urinary tract symptoms in men who underwent transurethral prostate resection. Asian J Androl 2017;19(02): 230-233

14 Higashi Y. Lower urinary tract symptoms/benign prostatic hypertrophy and vascular function: Role of the nitric oxide- phosphodiesterase type 5-cyclic guanosine 3',5'-monophosphate pathway. Int J Urol 2017;24(06):412-424

15 De Nunzio C, Presicce F, Tubaro A. Inflammatory mediators in the development and progression of benign prostatic hyperplasia. Nat Rev Urol 2016;13(10):613-626

16 Nickel JC, Roehrborn CG, Castro-Santamaria R, Freedland SJ, Moreira DM. Chronic Prostate Inflammation is Associated with Severity and Progression of Benign Prostatic Hyperplasia, Lower Urinary Tract Symptoms and Risk of Acute Urinary Retention. J Urol 2016;196(05):1493-1498 